\title{
Effects of Nutrients Foliar Application on Agrophysiological Characteristics of Maize under Water Deficit Stress
}

\author{
Nour Ali SAJEDI \\ Islamic Azad University, Faculty of Agriculture and Natural Resources, Arak, Iran; n-sajedi@iau-Arak.ac.ir
}

\begin{abstract}
To investigate effects of nutrients foliar application on agrophysiological characteristics of maize hybrid 'KSC 704' water deficit stress conditions, an experiment was arranged in a split plot factorial based on a randomized complete block design with four replications to the Research Station of Islamic Azad University-Arak Branch, Iran in 2007-2008. Main factors studied were four irrigation levels including irrigation equal to crop water requirement, water deficit stress at eight-leaf stage (V8), blister stage (R2) and filling grain stage (R4) in the main plot. Combined levels of selenium treatment (without and with application 20 gha $^{-1}$ ) and micronutrients (without and with application $2 \mathrm{lha}^{-1}$ ) were situated in sub plots. Results showed that water deficit stress decreased grain yield $19.7 \%$ in blister stage as compared with control. Using selenium increased relative content water at R2 and R4 stages significantly. Using selenium in water deficit stress condition increased measured traits except plant height as compared with treatment without selenium. A negative antagonistic interaction was found between selenium and micronutrients on some measured traits. Between treatments of water deficit stress, highest grain yield equal 6799.52 and $6736.97 \mathrm{kgha}^{-1}$ was obtained from combined treatments of water deficit stress at eight-leaf stage+without selenium+without micronutrients and water deficit stress at eight-leaf stage+selenium+without micronutrients respectively which compared with treatment of irrigation equal to crop water requirement+selenium+microelements did not differ significant. According to the results of experiment, it is concluded that with micronutrients fertilizer spray under optimum irrigation and selenium spray under water deficit obtain optimum yield.
\end{abstract}

Keywords: water deficit stress, selenium, relative content water, grain yield

\section{Introduction}

In many regions of the world, including Iran, drought stress is one of the most important factors that decrease agricultural crop production. Efficient water management will enhance the food supply in the coming decades (Yudelman, 1994). Proper management of inputs using modern technology, particularly irrigation water management and nutrient application is essential to maximize crop production and returns for the farmers (Panda et al., 2004). Water shortage during the vegetative stages of development limits the grain yield in many maize production areas. Pandey et al. (2000) reported that yield reduction (22.6-26.4\%) caused by deficit irrigation was associated with a decrease in kernel number and weight. Karam et al. (2003) stated that grain yield was reduced by $37 \%$ due to a decline of $18 \%$ in kernel weight and $10 \%$ in kernel number under water stress conditions.

Heavy metals, such as $\mathrm{Fe}, \mathrm{Cu}, \mathrm{Mn}$ and $\mathrm{Zn}$, function as micronutrients and are indispensable for plant growth (Haydon and Cobbett, 2007). Deficiencies of these heavy metals cause severe growth reduction (Broadley et al., 2007; Marschner, 1995). Several metabolic processes, such as photosynthesis, cellular respiration, nutrient uptake and photolysis of water, may be affected by the presence of microelements like $\mathrm{Zn}, \mathrm{Cu}, \mathrm{Fe}, \mathrm{Mn}$ and $\mathrm{Mo}$ (Sikora and Cieslik, 1999). Soils of Iran are deficient in organic matter. Similarly, most of micronutrients, for example Fe and $\mathrm{Mn}$ are readily fixed in soil having alkaline $\mathrm{pH}$. Plant roots are unable to absorb these nutrients adequately from dry topsoil (Foth and Ellis, 1996; Graham et al., 1992). Foliar spray of different micronutrients has been reported to be equally or more effective as soil application by different researchers (Torun et al., 2001).

Selenium is an essential micronutrient for animals, but its nutritional requirement in plants is not clearly understood. Selenium enrichment of crop plants results in the transformation of inorganic Se to bioavailability organic Se compounds within the plants (Gissel-Nielsen, 1994). In some plant species drought stimulates oxidation process which causes accumulation of poisonous oxygen such as free oxygen radical, hydrogen peroxide and hydroxyl radicals. Oxidative stress can prevent photosynthetic activity, respiration process and plant growth. Plants are naturally provided by enzymatic and non-enzymatic systems to take care active oxygen (Giang and Huang, 2001). Selenium may increase the tolerance of plant to UV-radiation induced oxidative stress, delay senescence and promote the 
40

growth of ageing seedling (Djanaguiraman et al., 2005). Result from Pennanen et al. (2002) has showed that plant growth promoted by selenium is due to increased starch accumulation in chloroplast. Recently, it has been shown that selenium can regulate the water status of plant under condition of water deficiency and thereby has a protective effect (Kuznetsov et al., 2003). The goal of this work was to determine the response on some agro physiological characteristics of maize to the combined effects water deficit, microelements and selenium foliar application.

\section{Materials and methods}

This experiment was conducted at Agricultural Research Station of Islamic Azad University, Arak Branch with $1779 \mathrm{~m}$ above sea level in Markazi province in center Iran in 2007 and 2008. The experimental design was split plot factorial based on randomized complete block design with four replications. Four irrigation levels including, without water stress (control), water deficit stress in Vegetative stage (V8), water deficit stress in blister stage (R2) and water deficit stress in grain filling stage (R4) were assigned to main plot. Water stress was executed by temporary stopping irrigation at each mentioned stages. In control treatment, irrigation conducted in equal crop water requirement. Crop water requirement was calculated by evaporation basin. Daily evaporation was measured from basin. Basin coefficient and crop coefficient was determined, amount of water consumed for each crop growth stage. Amount of water to plots was controlled by water contour. Irrigation of again in stress treatments were done when soil moisture arrived at 15\% weight of soil. Microelements at two levels, without and with using that was provided by specific fertilizer for maize called " biomin "which contains microelements of $\mathrm{Fe}, \mathrm{Zn}, \mathrm{Cu}$, $\mathrm{Mn}, \mathrm{B}, \mathrm{Mo}$ and $\mathrm{Mg}$ in the form of foliar application at sixleaf stage and one week before tassling stage at the rate of $2 \mathrm{lha}^{-1}$. Selenium as sodium selenite $\left(\mathrm{NaHSeO}_{3}\right)$ at two levels (with and without) was applied two weeks before execution of water stress treatment at the rate of $20 \mathrm{gha}^{-1}$. Microelements and selenium were situated in sub plots as randomized. Soil preparation including plow was done in fall and perpendicular disks each two years. Each plot constituted seeded liners distanced $65 \mathrm{~cm}$ from each other and $20 \mathrm{~cm}$ distance between each two plants on the lines. The length of each seeded line was six meters and two furrows between each two plots were unseeded. Maize seeds (hybrid single cross 704) were hand-seeded on $18^{\text {th }}$ of May each two years. A mixed sample of soil 0-30 cm deep was prepared to determine the EC of the saturated paste (Allison and Moodie, 1965) $\mathrm{pH}$, texture with hydrometer (Bouyoucos, 1962), organic C percentage (Walkey and Black, 1934), total N (Kjeldahl method), the concentration of available $\mathrm{P}$ (Olsen et al., 1954) and available $\mathrm{K}$ with flame (U.S. Salinity Laboratory, 1954). Iron, Zn, Mn and $\mathrm{Cu}$ were determined with method of diethylenetriamine- pentaacetic acid (DTPA) (Lindsay and Norvell, 1979). All treatments received $187.5 \mathrm{kgha}^{-1} \mathrm{~N}$ as urea, $150 \mathrm{kgha}^{-1}$ $\mathrm{P}_{2} \mathrm{O}_{5}$ as super phosphate triple and $150 \mathrm{~K}_{2} \mathrm{O}$ as sulphate potasium in each year. One third of $\mathrm{N}$ and total rate of $\mathrm{P}_{2} \mathrm{O}_{5}$ and $\mathrm{K}_{2} \mathrm{O}$ were applied before planting. The remaining $\mathrm{N}$ was applied in equal rate at six-leaf stage and two week before tassling stage.

Relative water content was calculated to examine plant reaction to water deficit stress. For this purpose, leaves of three plants from second and fifth rows were separated before irrigation at $12 \mathrm{pm}$ and were taken to the laboratory. In the laboratory disks of the leaves were prepared and weighed immediately to measure the fresh leaf weight. Then the disks were placed in distilled water for about 24 hours at $4^{\circ} \mathrm{C}$ until they were saturated completely. At the end of this stage, the leaf disks were dried by towels of dry paper and were then weighed again. The samples were placed in the oven for about 48 hours at $72^{\circ} \mathrm{C}$ until they were dried. The weight of dried leaves was recorded. Relative water content was calculated using the following relation (Dhopte and Manuel, 2002)

$$
R W C=\frac{W f-W d}{W s-W d} \times 100
$$

RWC=Relative Water Content

Wf $=$ Fresh Leaf Weight

Wd=Dry Leaf Weight

Ws=Saturated Leaf Weight

Final harvest was performed at physiological maturity stage when a black layer was formed at seed base. At maturity stage, grain yield was determined from a harvest area of $1.5 \times 4 \mathrm{~m}$ ( 2 rows middle of each experimental plot) and expressed on a $15 \%$ moisture basis.

To determine water use efficiency the following relation was applied:

Water use economic efficiency $=$ Grain yield $\left(\mathrm{kgha}^{-1}\right) /$ water used $\left(\mathrm{m}^{3} \mathrm{ha}^{-1}\right)$

Analysis of variance was performed using PROC ANOVA of SAS. The comparison of the means was done by Duncan's test at a probability level of 5 percent.

\section{Results and discussions}

Effects of water deficit stress on Plant height, grain yield, relative content water at V8, R2 and R4 stages was significant at $1 \%$ level (Tab. 2). Mean comparison of treatments indicate that highest rate of grain yield, relative content Water at V8, R2 and R4 stages related to treatment of without water deficit stress and highest rate of water use efficiency obtained from treatment of water deficit stress in V8 stage (Tab. 3). Water deficit stress decreased Plant height $14.4 \%$ in stage V8 as compared with control. It is known that a decrease in plant height is due to a decrease in cell division and assimilate transport. There are many reports of a decrease of vegetative growth and plant height under conditions of drought stress. An increase in plant 
Tab. 1. Result of physical and chemical soil analysis

\begin{tabular}{|c|c|c|c|c|c|c|c|c|c|c|c|c|c|c|c|}
\hline Year & $\begin{array}{c}\text { Depth } \\
(\mathrm{cm})\end{array}$ & $\begin{array}{c}\text { EC } \\
\text { ds } \mathrm{m}^{-1}\end{array}$ & $\mathrm{PH}$ & OC $\%$ & N\% & $\begin{array}{c}\mathrm{P} \\
(\mathrm{ppm})\end{array}$ & $\begin{array}{c}\mathrm{K} \\
(\mathrm{ppm})\end{array}$ & $\begin{array}{c}\mathrm{Zn} \\
(\mathrm{ppm})\end{array}$ & $\begin{array}{c}\mathrm{Fe} \\
(\mathrm{ppm})\end{array}$ & $\begin{array}{c}\mathrm{Mn} \\
(\mathrm{ppm})\end{array}$ & $\begin{array}{c}\mathrm{Cu} \\
(\mathrm{ppm})\end{array}$ & $\begin{array}{c}\mathrm{Se} \\
(\mathrm{ppm})\end{array}$ & $\begin{array}{c}\text { Sand } \\
\%\end{array}$ & $\begin{array}{c}\text { Silt } \\
\% \\
\end{array}$ & $\begin{array}{c}\text { Clay } \\
\%\end{array}$ \\
\hline 2007 & $0-30$ & 1.20 & 7.5 & 0.82 & 0.08 & 5 & 150 & 0.8 & 4.6 & 10.6 & 1.14 & 027 & 29 & 35 & 36 \\
\hline 2008 & $0-30$ & 1.70 & 7.7 & 0.78 & 0.06 & 8.5 & 130 & 9.2 & 3.9 & 9.2 & 1.1 & 0.23 & 26 & 38 & 36 \\
\hline
\end{tabular}

Tab. 2. Combined analysis of variance for traits of maize in water deficit stress, selenium and microelements (2008-2009)

\begin{tabular}{|c|c|c|c|c|c|c|c|}
\hline \multicolumn{8}{|c|}{ MS } \\
\hline S.O.V & $\mathrm{df}$ & Plant height & $\mathrm{RWC}_{\mathrm{V} 8}$ & $\mathrm{RWC}_{\mathrm{R} 2}$ & $\mathrm{RWC}_{\mathrm{R} 4}$ & WUE & Grain yield \\
\hline $\operatorname{Year}(\mathrm{Y})$ & 1 & $9154.52^{* *}$ & $2368.87^{* *}$ & $17.70 \mathrm{~ns}$ & $630.25^{* *}$ & $0.483^{*}$ & $14083664.10^{*}$ \\
\hline Replication (Year) & 6 & 438.87 & 138.60 & 28.61 & 31.05 & 0.089 & 2679445.25 \\
\hline Irrigation(I) & 3 & $4395.80^{* *}$ & $1615.42^{* *}$ & $265.97^{* *}$ & $596.86^{* *}$ & $0.067 \mathrm{~ns}$ & $8391972.81^{* *}$ \\
\hline$Y \times I$ & 3 & $793.82 \mathrm{~ns}$ & $148.59^{*}$ & $220.53^{* *}$ & $663.21^{* *}$ & $0.114^{*}$ & $11730544.18^{* *}$ \\
\hline Error & 18 & 315.67 & 80.41 & 21.01 & 68.47 & 0.027 & 115388.32 \\
\hline Selenium(Se) & 1 & $123.59 \mathrm{~ns}$ & $0.327 \mathrm{~ns}$ & $229.22^{* *}$ & $266.77^{* *}$ & $0.005 \mathrm{~ns}$ & $114990.06 \mathrm{~ns}$ \\
\hline Sex Y & 1 & 146.59ns & $29.27 \mathrm{~ns}$ & $90.23 \mathrm{~ns}$ & $13.05 \mathrm{~ns}$ & $0.011 \mathrm{~ns}$ & $214411.12 \mathrm{~ns}$ \\
\hline Sex I & 3 & $42.55 \mathrm{~ns}$ & $166.02^{*}$ & $100.12^{*}$ & $58.87 \mathrm{~ns}$ & $0.093^{* *}$ & $6653116.13^{* *}$ \\
\hline Se $\times I \times Y$ & 3 & $202.66 \mathrm{~ns}$ & $213.25^{* *}$ & $18.43^{*}$ & $93.32^{*}$ & $0.109^{* *}$ & $6920361.48^{* *}$ \\
\hline Microelement (M) & 1 & $776.67 \mathrm{~ns}$ & 73.40 & $266.48^{* *}$ & $42.67 \mathrm{~ns}$ & $0.062 \mathrm{~ns}$ & $3628562.64^{*}$ \\
\hline$M \times Y$ & 1 & $177.09 \mathrm{~ns}$ & $0.654 \mathrm{~ns}$ & $0.404 \mathrm{~ns}$ & $5.37 \mathrm{~ns}$ & $0.001 \mathrm{~ns}$ & $17021.52 \mathrm{~ns}$ \\
\hline $\mathrm{I} \times \mathrm{M}$ & 3 & $286.68^{*}$ & $39.62 \mathrm{~ns}$ & $23.38 \mathrm{~ns}$ & $171.08^{* *}$ & $0.048^{*}$ & $3250076.38^{* *}$ \\
\hline $\mathrm{Y} \times \mathrm{I} \times \mathrm{M}$ & 3 & $338.53^{*}$ & $57.08 \mathrm{~ns}$ & $41.54 \mathrm{~ns}$ & $122.19^{* *}$ & $0.006 \mathrm{~ns}$ & $438666.77 \mathrm{~ns}$ \\
\hline Se $\times M$ & 1 & $809.32^{* *}$ & $191.17^{*}$ & $2.16 \mathrm{~ns}$ & $8.02 \mathrm{~ns}$ & $0.011 \mathrm{~ns}$ & 742736.48ns \\
\hline $\operatorname{Se} \times M \times Y$ & 1 & $179.31 \mathrm{~ns}$ & $1.38 \mathrm{~ns}$ & $90 / 100 \mathrm{~ns}$ & $53.88 \mathrm{~ns}$ & $0.114^{* *}$ & $6558182.18^{* *}$ \\
\hline $\operatorname{Se} \times M \times I$ & 3 & $76.31 \mathrm{~ns}$ & $139.92^{*}$ & ${ }^{* *} 05 / 230$ & $136.36^{* *}$ & $0.013 \mathrm{~ns}$ & $691369.35^{* *}$ \\
\hline $\mathrm{Se} \times \mathrm{M} \times \mathrm{I} \times \mathrm{Y}$ & 6 & $198.86 \mathrm{~ns}$ & $77.98 \mathrm{~ns}$ & $11.33 \mathrm{~ns}$ & ${ }^{* *} 145.57$ & $0.064^{*}$ & $3361080.25^{* *}$ \\
\hline Error & 72 & 94.94 & 41.88 & 26.94 & 25.55 & 0.016 & 710068.41 \\
\hline C.V (\%) & & 7.12 & 9.29 & 6.51 & 6.72 & 15.23 & 13.06 \\
\hline
\end{tabular}

Tab. 3. Mean comparisons plant characteristics of maize in main effects of year, irrigation, selenium and interaction of selenium $\times$ irrigation

\begin{tabular}{|c|c|c|c|c|c|c|}
\hline Treatments & $\begin{array}{c}\text { Plant height } \\
(\mathrm{cm})\end{array}$ & $\begin{array}{c}\mathrm{RWC}_{\mathrm{v} 8} \\
(\%)\end{array}$ & $\begin{array}{c}\mathrm{RWC}_{\mathrm{R} 2} \\
(\%)\end{array}$ & $\begin{array}{c}\mathrm{RWC}_{\mathrm{R} 4} \\
(\%)\end{array}$ & $\begin{array}{c}\text { WUE } \\
\left(\mathrm{kg} \cdot \mathrm{m}^{-3}\right)\end{array}$ & $\begin{array}{c}\text { Grain yield } \\
\left(\mathrm{kg} \cdot \mathrm{ha}^{-1}\right)\end{array}$ \\
\hline \multicolumn{7}{|c|}{ Years } \\
\hline 2007 & $145.35 a$ & $73.93 \mathrm{a}$ & $79.37 \mathrm{a}$ & $77.01 \mathrm{a}$ & $0.89 \mathrm{a}$ & $6781.55 a$ \\
\hline 2008 & $128.41 \mathrm{~b}$ & $65.33 b$ & $80.12 \mathrm{a}$ & $77.45 b$ & $0.75 b$ & $6118.14 b$ \\
\hline \multicolumn{7}{|c|}{ Irrigation } \\
\hline I0 & $139.63 a$ & $74.11 \mathrm{a}$ & $82.67 \mathrm{a}$ & $77.90 \mathrm{a}$ & $0.84 \mathrm{ab}$ & $7076.55 a$ \\
\hline I1 & $119.59 \mathrm{~b}$ & $59.06 \mathrm{~b}$ & $80.28 \mathrm{a}$ & $77.70 \mathrm{a}$ & $0.87 \mathrm{a}$ & $6603.75 \mathrm{ab}$ \\
\hline I2 & $144.15 a$ & $73.45 \mathrm{a}$ & $75.60 \mathrm{~b}$ & $76.50 \mathrm{a}$ & $0.75 b$ & $5702.05 c$ \\
\hline $\mathrm{I} 3$ & $144.14 a$ & $71.89 \mathrm{a}$ & $80.42 \mathrm{a}$ & $68.82 \mathrm{~b}$ & $0.77 b$ & $5878.14 \mathrm{bc}$ \\
\hline \multicolumn{7}{|c|}{ Selenium } \\
\hline $\mathrm{Se} 0$ & $137.86 \mathrm{a}$ & $69.58 \mathrm{a}$ & $78.41 \mathrm{~b}$ & $73.79 b$ & $0.83 \mathrm{a}$ & $6419.87 \mathrm{a}$ \\
\hline Sel & $135.90 \mathrm{a}$ & $69.68 \mathrm{a}$ & $81.08 \mathrm{a}$ & $76.67 \mathrm{a}$ & $0.84 \mathrm{a}$ & $6479.82 \mathrm{a}$ \\
\hline \multicolumn{7}{|c|}{ Irrigation $\times$ Selenium } \\
\hline I0 SeO 0 & $141.81 \mathrm{ab}$ & $76.66 \mathrm{a}$ & $80.19 \mathrm{~b}$ & $77.32 \mathrm{a}$ & $0.92 \mathrm{a}$ & $7656.73 a$ \\
\hline I0 Sel & $137.46 \mathrm{~b}$ & $71.55 \mathrm{bc}$ & $81.15 \mathrm{ab}$ & $78.48 \mathrm{a}$ & $0.79 b c$ & $6616.50 \mathrm{~b}$ \\
\hline Il Se0 & $120.51 \mathrm{c}$ & $57.99 \mathrm{~d}$ & $81.52 \mathrm{ab}$ & 76.18ab & $0.88 \mathrm{ab}$ & $6496.14 b$ \\
\hline I1Sel & $118.67 \mathrm{c}$ & $60.14 d$ & $83.04 \mathrm{ab}$ & $79.22 \mathrm{a}$ & $0.88 \mathrm{ab}$ & $6591.00 \mathrm{~b}$ \\
\hline $\mathrm{I} 2 \mathrm{Se} 0$ & $143.59 \mathrm{ab}$ & $74.42 \mathrm{ab}$ & $75.45 c$ & $73.17 \mathrm{~b}$ & $0.80 \mathrm{bc}$ & $6019.26 \mathrm{~b}$ \\
\hline I2 Sel & $144.72 \mathrm{ab}$ & $72.49 \mathrm{abc}$ & $75.75 c$ & $79.84 a$ & $0.85 \mathrm{ab}$ & $6462.83 \mathrm{~b}$ \\
\hline $\mathrm{I} 3 \mathrm{Se} 0$ & $145.55 a$ & $69.25 c$ & $76.45 c$ & $68.47 \mathrm{c}$ & $0.71 \mathrm{c}$ & $5386.99 \mathrm{c}$ \\
\hline I3 Sel & $142.73 \mathrm{ab}$ & 74.53ab & $84.38 \mathrm{a}$ & $69.16 \mathrm{c}$ & $0.83 \mathrm{ab}$ & $6369.29 \mathrm{~b}$ \\
\hline
\end{tabular}


42

Tab. 4. Mean comparisons plant characteristics of maize in main effects of microelement fertilizer, interaction of irrigation $\times$ microelement fertilizer and selenium $\times$ microelement fertilizer

\begin{tabular}{|c|c|c|c|c|c|c|}
\hline Treatments & $\begin{array}{c}\text { Plant height } \\
(\mathrm{cm})\end{array}$ & $\begin{array}{c}\mathrm{RWC}_{\mathrm{v} 8} \\
(\%)\end{array}$ & $\begin{array}{c}\mathrm{RWC}_{\mathrm{R} 2} \\
(\%)\end{array}$ & $\begin{array}{c}\mathrm{RWC}_{\mathrm{R} 4} \\
(\%)\end{array}$ & $\begin{array}{c}\text { WUE } \\
\left(\mathrm{kg} \cdot \mathrm{m}^{-3}\right)\end{array}$ & $\begin{array}{c}\text { Grain yield } \\
\left(\mathrm{kg} . \mathrm{ha}^{-1}\right)\end{array}$ \\
\hline \multicolumn{7}{|c|}{ Microelement } \\
\hline M0 & $139.34 \mathrm{a}$ & $68.87 \mathrm{a}$ & $78.30 \mathrm{~b}$ & $75.81 \mathrm{a}$ & $0.85 \mathrm{a}$ & $6618.21 \mathrm{a}$ \\
\hline M1 & $134.42 \mathrm{a}$ & $70.39 a$ & $81.19 \mathrm{a}$ & $74.60 \mathrm{a}$ & $0.81 \mathrm{a}$ & $6281.47 \mathrm{~b}$ \\
\hline \multicolumn{7}{|c|}{ Microelement $\times$ Irrigation } \\
\hline M0 I0 & $139.14 \mathrm{bc}$ & $74.97 \mathrm{a}$ & $78.79 \mathrm{bc}$ & $80.56 a$ & $0.83 \mathrm{a}$ & $6850.14 \mathrm{ab}$ \\
\hline M1 I0 & $150.13 a$ & $73.25 \mathrm{a}$ & $82.55 \mathrm{ab}$ & $75.23 b c$ & $0.88 \mathrm{a}$ & $7320.73 a$ \\
\hline M0 I1 & $121.34 \mathrm{~d}$ & $57.75 b$ & $79.93 \mathrm{bc}$ & $78.11 \mathrm{ab}$ & $0.90 \mathrm{a}$ & $6768.25 \mathrm{ab}$ \\
\hline M1 I1 & $117.83 \mathrm{~d}$ & $60.38 b$ & $84.63 a$ & $78.29 \mathrm{ab}$ & $0.86 a$ & $6439.25 b c$ \\
\hline M0 I2 & $140.78 b c$ & $72.49 \mathrm{a}$ & $74.46 \mathrm{~d}$ & $73.91 \mathrm{~cd}$ & $0.85 a$ & $6422.27 b c$ \\
\hline M1 I2 & $137.52 \mathrm{c}$ & $74.41 \mathrm{a}$ & $76.74 \mathrm{~cd}$ & $79.10 \mathrm{a}$ & $0.80 \mathrm{a}$ & $6059.82 \mathrm{c}$ \\
\hline M0 I3 & $146.10 \mathrm{ab}$ & $70.28 \mathrm{a}$ & $80.02 \mathrm{bc}$ & $70.65 \mathrm{~d}$ & $0.84 \mathrm{a}$ & $6432.19 b c$ \\
\hline M1 I3 & $142.18 b c$ & $73.5 \mathrm{a}$ & $80.82 \mathrm{ab}$ & $70.99 \mathrm{~d}$ & $0.70 \mathrm{~b}$ & $5324.09 \mathrm{~d}$ \\
\hline \multicolumn{7}{|c|}{ Microelement $\times$ Selenium } \\
\hline M0 Se0 & $142.84 a$ & $67.60 \mathrm{~b}$ & $77.09 \mathrm{~b}$ & 74.61ab & $0.86 a$ & $6664.41 \mathrm{a}$ \\
\hline M1 Se0 & $132.88 \mathrm{~b}$ & $70.14 a b$ & $79.72 b$ & $72.96 \mathrm{~b}$ & $0.79 \mathrm{a}$ & $6175.33 b$ \\
\hline M0 Sel & $135.85 \mathrm{~b}$ & $71.56 a$ & $79.51 b$ & $77.00 \mathrm{a}$ & $0.85 a$ & $6572.01 \mathrm{ab}$ \\
\hline M1 Sel & $135.95 \mathrm{~b}$ & 69.21ab & $82.65 \mathrm{a}$ & $76.35 a$ & $0.83 a$ & $6387.62 \mathrm{ab}$ \\
\hline
\end{tabular}

Means in each followed by the similar letters are not significantly different at $5 \%$ probability level using Duncan, Multiple Range test

height is related to two phenomena, an increase in node number and an increase of inter-node length and these are strongly affected by drought stress (Wright et al., 1988). Water deficit stress decreased grain yield 6\% in stage V8, $19.7 \%$ in stage of blister and $16.9 \%$ in stage of grain filling as compared with control. Decrease in grain yield can due to reduced leaf, silk and grain kernel expansion, re- duced assimilate flux to growing organs, accelerated leaf senescence, delayed silk growth and greater ear and kernel abortion (Bassetti and Westgate, 1993). Also result of this research is parallel with results of other researchers (Bassetti and Westgate, 1993; Schussler and Westgate, 1995).

Effect of selenium on relative content Water at R2 and R4 stages was significant but on relative content Water

Tab. 5. Mean comparisons of plant characteristics of maize in interaction effects of threefold irrigation $\times$ selenium microelements fertilizer treatments

\begin{tabular}{|c|c|c|c|c|c|c|c|c|}
\hline Irrigation & Selenium & Microelement & $\begin{array}{c}\text { Plant height } \\
(\mathrm{cm})\end{array}$ & $\begin{array}{c}\mathrm{RWC}_{\mathrm{v} 8} \\
(\%)\end{array}$ & $\begin{array}{c}\mathrm{RWC}_{\mathrm{R} 2} \\
(\%)\end{array}$ & $\begin{array}{c}\mathrm{RWC}_{\mathrm{R} 4} \\
(\%)\end{array}$ & $\begin{array}{c}\text { WUE } \\
\left(\mathrm{kg} \cdot \mathrm{m}^{-3}\right)\end{array}$ & $\begin{array}{c}\text { Grain yield } \\
\left(\mathrm{kg} . \mathrm{ha}^{-1}\right)\end{array}$ \\
\hline I0 & $\mathrm{Se} 0$ & M0 & $143.36 \mathrm{ab}$ & $78.40 \mathrm{a}$ & $77.60 c-f$ & $81.27 \mathrm{a}$ & $\mathrm{abc} 88 / 0$ & $7336.55 \mathrm{ab}$ \\
\hline I0 & $\mathrm{Se} 0$ & M1 & $140.25 \mathrm{ab}$ & $74.93 \mathrm{abc}$ & $82.79 \mathrm{abc}$ & $73.36 \mathrm{cde}$ & $0.96 \mathrm{a}$ & $7976.91 \mathrm{a}$ \\
\hline I0 & Sel & M0 & $134.92 b c$ & $71.55 \mathrm{abc}$ & $79.99 \mathrm{~b}-\mathrm{e}$ & 79.86ab & $0.77 \mathrm{bc}$ & $6790.73 b c$ \\
\hline I0 & Sel & M1 & $140.01 \mathrm{ab}$ & $71.56 \mathrm{abc}$ & $82.32 \mathrm{abc}$ & $77.10 \mathrm{abc}$ & $0.81 \mathrm{bc}$ & $6628.55 \mathrm{bc}$ \\
\hline I1 & $\mathrm{Se} 0$ & M0 & $126.16 \mathrm{~cd}$ & $53.33 \mathrm{f}$ & 75.96def & $78.04 a b c$ & $0.90 \mathrm{ab}$ & $6799.52 \mathrm{bc}$ \\
\hline I1 & $\mathrm{Se} 0$ & M1 & $114.86 \mathrm{e}$ & $62.65 \mathrm{de}$ & $87.09 \mathrm{a}$ & $74.33 \mathrm{bcd}$ & $0.86 \mathrm{abc}$ & $64.33 .48 \mathrm{bcd}$ \\
\hline I1 & Sel & M0 & $116.53 \mathrm{e}$ & $62.16 \mathrm{de}$ & 83.91ab & $78.18 \mathrm{abc}$ & $0.90 \mathrm{ab}$ & $6736.97 \mathrm{bc}$ \\
\hline I1 & Sel & M1 & $120.82 \mathrm{de}$ & $58.11 \mathrm{ef}$ & $82.18 \mathrm{abc}$ & $80.25 \mathrm{a}$ & $0.85 \mathrm{abc}$ & $6445.03 \mathrm{bcd}$ \\
\hline I2 & $\mathrm{Se} 0$ & M0 & $150.76 \mathrm{a}$ & $70.79 \mathrm{bc}$ & 75.88def & $67.74 \mathrm{fg}$ & $0.85 \mathrm{abc}$ & 6448.16bcd \\
\hline I2 & $\mathrm{Se} 0$ & M1 & $136.42 b$ & $78.04 a b$ & $75.03 \mathrm{ef}$ & 78.61abc & $074 \mathrm{~cd}$ & $5590.36 \mathrm{~d}$ \\
\hline I2 & Sel & M0 & $151.81 \mathrm{a}$ & $74.20 \mathrm{abc}$ & $73.05 f$ & $80.08 \mathrm{ab}$ & $0.85 \mathrm{abc}$ & $6396.38 \mathrm{bcd}$ \\
\hline I2 & Sel & M1 & $138.62 b$ & $70.79 \mathrm{bc}$ & $78.45 b-f$ & 79.59ab & $0.86 \mathrm{abc}$ & $6529.28 \mathrm{bcd}$ \\
\hline I3 & $\mathrm{Se} 0$ & M0 & $151.08 \mathrm{a}$ & $67.88 \mathrm{~cd}$ & $78.94 b-f$ & $71.41 \mathrm{def}$ & $0.80 \mathrm{bc}$ & $6073.43 \mathrm{~cd}$ \\
\hline $\mathrm{I} 3$ & $\mathrm{Se} 0$ & M1 & $140.01 \mathrm{ab}$ & $70.62 b c$ & $73.96 f$ & $65.53 \mathrm{~g}$ & $0.63 \mathrm{~d}$ & $4700.55 \mathrm{e}$ \\
\hline I3 & Sel & M0 & 141.12ab & $72.67 \mathrm{abc}$ & $81.09 \mathrm{bcd}$ & $69.89 \mathrm{~d}-\mathrm{g}$ & $0.88 \mathrm{abc}$ & 6363.96bcd \\
\hline $\mathrm{I} 3$ & Sel & M1 & $144.35 \mathrm{ab}$ & 76.39ab & $87.67 \mathrm{a}$ & $68.44 \mathrm{efg}$ & $0.78 b c$ & $5947.62 \mathrm{~cd}$ \\
\hline
\end{tabular}

Means in each followed by the similar letters are not significantly different at $5 \%$ probability level using Duncan, Multiple Range test 
at V8, water use efficiency was and grain yield not significant, however with using selenium mentioned traits, were increased (Tab. 3). This is important from viewpoint of quantity and quality for consumption human and animals. Result of this research is parallel with results of Pennanen $e t$ al. (2002), those reported that plant growth promoted by selenium is due to increased starch accumulation in chloroplast. Selenium seemed to improve pollen survival and fertilization (Seppnen et al., 2003). Xue et al. (2001) showed that enhancement of photosynthesis and a decrease in leaf senescence increases assimilate production and towards seed and as result seed yield increases.

Results of two fold interactions water deficit stress and selenium on relative content Water at V8 and R2 stages, water use efficiency and grain yield was significant but on Plant height and relative content Water at V4 not significant (Tab. 2). Using selenium both in water optimum and water deficit stress increased relative content Water at R2 and R4 stages (Tab. 3). Selenium seemed that can regulate the water status of plant under condition of water deficiency and thereby has a protective effect of water stress (Kuznetsov et al., 2003). Highest grain yield (7656.73 kgha $^{-1}$ ) was obtained from control treatment (Without stress and without Selenium) which showed significant differences in compared to other treatments. Using selenium in water deficit stress condition increased all of traits except plant height as compared to treatment without using selenium (Tab. 3). It has been reported that selenium prevents chlorophyll degradation under stress conditions (Seppnen et al., 2003).

It has been reported that selenium has antioxidant properties and under conditions of environmental stress, especially water stress, it can scavenge reactive oxygen species (Xue et al., 2001). Glutathione proxidase is an important enzyme in antioxidant defense systems of plant. Selenium is an essential element in the structure of this enzyme (Timothy, 2001). Least grain yield was obtained from treatment of water deficit stress in grain filling stage and without Selenium application (Tab. 3). This might be indicative of plant sensitivity due to no protection factor under water stress.

Application of microelements decreased grain yield significantly at $5 \%$ level (Tab. 1). This may be related to antagonistic interaction of microelements to each other. In maize, with copper application alone and along with iron and manganese decreased grain yield (Himayatullah and Khan, 1998).

Using microelements in water optimum conditions increased traits measured as compared to without microelements but in water deficit stress conditions in all growth stages, traits of measured were decreased. Using microelements in water deficit stress condition increased traits of relative content water at V8, R2 and R4 stages but traits of water use efficiency, plant height and grain yield decreased as compared to treatment without using microelements
(Tab. 4). Presumable in water deficit stress condition was decreased remobilization microelements.

Three fold interactions experimental factors on relative content water at V8, R2 and R4 stages was significant but on water use efficiency, plant height and grain yield was not significant. Using of along selenium and microelements under water deficit stress conditions in vegetative stage (V8) and reproductive stage (R2 and R4 stage) increased relative content water at three stages in compared to without using this elements in water stress conditions (Tab. 5). The highest amounts of grain yield (7976.91 kgha ${ }^{-1}$ obtain from treatment of water optimum conditions+without selenium+with microelements (Tab. 5). Using selenium and microelements under water deficit stress conditions in blister stage (R2) increased grain yield in compared to without using this elements in water stress conditions (Tab. 5). Presumable in the end of growth period that increased stress conditions, and decrease mechanism of defensive plant, selenium and microelements with take part in biological activity of cells, induced health protection and permanent in function of membranes.

\section{Conclusions}

Improvements in maize drought tolerance are therefore vital for maintaining local and global food security. In general, plants tolerated unfavorable environmental conditions by changing their morphology as living indices. Results show that selenium foliar applications can improve yield under conditions of drought stress and it may be recommended for lands in arid semi- arid regions. Other microelements such as $\mathrm{Fe}, \mathrm{Zn}, \mathrm{Cu}, \mathrm{Mg}$ and Mn also play their role as cofactors in biological activities the structure of plant therefore when plants are deficient of these elements, increased sensitivity to environmental stresses. Using microelements in water optimum condition increased traits of measured as compared to without microelements but in water deficit stress condition in all growth stages traits were decreased. Using of selenium and microelements in water deficit stress condition in vegetative growth stage and dough stage increased yield as compared to without using this elements in water stress condition. The combined results of these studies indicate that by using selenium and microelements under water stress can produce suitable quantitative and qualitative as compared to without using this elements.

\section{References}

Allison, L. E. and C. D. Moodie (1965). Carbonate, p. 13791396. In: Black C. A. Eds. Methods of soil nalysis, part II. American Society of Agronomy Madison, Wisc.

Bassetti, P. and M. E. Westgate (1993). Water deficit affects receptivity of maize silks. Crop Science 33:279-282.

Bouyoucos, C. J. (1962). Hydrometer method for making particle-size analysis of soils. Agronomy Journal 57:464- 
44

465.

Broadley, M., R. White, P. J. Hammond, J. P. Zelko and I. Lux (2007). Zinc in plants. New Phytol. 173:677-702.

Dhopte, A. M. and L. M. Manuel (2002). Principals and techniques for plant scientists. Lst End. Updesh purohit for Agrobios(India). Odupur 373.

Djanaguiraman, M., D. D. Devi, A. K. Shanker, A. Sheeba and U. Bangarusamy (2005). Selenium - an antioxidative protectant in soybean during senescence. Plant and Soil 272:77-86.

Foth, H. D. and B. G. Ellis (1996). Soil fertility. 2nd Ed. Lewis Pub. New York.

Giang, Y. and B. Huang (2001). Drought and heat stress injury two cool- season turf grasses in relation to antioxidant metabolism and lipid peroxidation. Crop Science 41:436442.

Graham, R. D., J. S. Ascher and S. C. Hynes (1992). Selecting zinc-efficient cereal genotypes for soils of low zinc status. Plant and Soil 146:241-250.

Himayatullah, B. and M. Khan (1998). Response of irrigated maize to trace elements in the presence of NPK sarhad. Journal Agric. 14:117-120.

Haydon, M. J. and C. S. Cobbett (2007). Transporters of ligands for essential metal ions in plants. New Phytol. 174:499-506.

Karam, F., J. Breidy, C. Stephan and J. Rouphael (2003). Evapotranspiration, yield and water use efficiency of drip irrigated corn in the Bekaa Valley of Lebanon, Agric. Water Manage. 63(2):125-137.

Kuznetsov, V. V., V. P. Kholodova and B. A. Yagodin (2003). Selenium regulates the water status of plants exposed to drought. Dokl. Biol. Sci. 390:266-268.

Lindsay, W. L. and W. A. Norvell (1979). Development of a DTPA test for zinc, iron, manganese, and copper. Soil Science Society of America Journal 42:421-428.

Marschner, H. (1995). Mineral Nutrition of Higher Plants, 2nd edn. Academic Press, London (1995) p. 889.

Panda, R. K., S. K. Behera and P. S. Kashyap (2004). Effective management of irrigation water for maize under stressed conditions, Agricultural Water Management 66(3):181203.

Pandey, R. K., J. W. Maranville and A. Admou (2000). Deficit irrigation and nitrogen effects on maize in a Sahelian environment. I. Grain yield and yield components, Agric. Water Manage. 46:1-13.

Pennanen, A., T. Xue and H. Hartikainen (2002). Protective role of selenium in plant subjected to severe UV irradiation stress. Journal of Applied Botany 76:66-76.

Schussler, J. R. and M. E. Westgate (1995). Assimilate flux determines kernel set at low water potential in maize. Crop Science 35:1074-1080.

Seppnen, M., M. Turkainen and H. Hartikainen (2003). The effect of selenium on photooxidative stress tolerance in potato. Plant Science 165: 311-319.

Sikora, E. and E. Cieslik (1999). Correlation between the levels of nitrates and nitrites and the contents of iron, copper and manganese in potato tubers, Food Chem. 67(3):301-304.

Timothy, P. (2001). Glutation-related enzymes and selenium status: implications for oxidative stress. Biochem Pharm 62:273-281.

Torun, A., I. G. Ã. Itekin, M. Kalayci, A. Yilmaz, S. Eker and I. Cakmak (2001). Effects of zinc fertilization on grain yield and shoot concentrations of zinc, boron, and phosphorus of 25 wheat cultivars grown on a zinc-deficient and borontoxic soil. Journal of Plant Nutrition 24(11):1817-1829.

U.S. Salinity Laboratory (1954). Diagnosis and improvement of saline and alkali soils (USDA Handbook No. 60).

Xue, T., H. Hartikainen and V. Piironen (2001). Antioxidative and growth- promoting effect of selenium in senescing lettuce. Plant and Soil 27:55-61.

Walkey, A. and T. A. Black (1934). An examination of the Dehlijaroff method for determining organic matter and a proposed modification of the chromic acid titration method. Soil Science 37:29-38.

Wright, G. C., C. J. Smith and M. R. Woodroofe (1988). The effect of irrigation and nitrogen fertilizer on rapeseed production in south-eastern Australia. Irrig. Sci. 9:1-13.

Yudelman, M. (1994). Feeding the world, Int. Irrig. Manage. Institute Rev. 8(1):4-15. 